\title{
Mapping Evidence on Management of Cervical Cancer in Sub-Saharan Africa: Scoping Review Protocol
}

Petmore Zibako ( $\sim$ petmoreword@yahoo.com )

University of Kwazulu-Natal

Mbuzeleni Hlongwa

University of KwaZulu-Natal College of Health Sciences

Nomsa Tsikai

University of Zimbabwe College of Health Sciences

Sarah Manyame

University of Zimbabwe College of Health Sciences

Themba G Ginindza

University of KwaZulu-Natal College of Health Sciences

\section{Protocol}

Keywords: cervical cancer management, control, prevention, screening, diagnosis, HPV vaccine, treatment, surgery, chemotherapy, radiotherapy, Sub-Saharan Africa

Posted Date: April 22nd, 2020

DOl: https://doi.org/10.21203/rs.3.rs-23265/v1

License: (1) This work is licensed under a Creative Commons Attribution 4.0 International License. Read Full License 


\section{Abstract}

\section{Background}

Cancer is a non-communicable disease, and is the number two leading cause of death globally. Among all cancers, cervical cancer is the number one killer of women in Low-Income countries (LICs). Cervical cancer is a well understood preventable cancer. The rates of cervical cancer are very varied and inversely proportional to effectiveness of disease management policies. Management of cervical cancer includes: prevention, screening, diagnosis and treatment. The main objective of this scoping review is to map evidence on cervical cancer management in sub-Saharan Africa (SSA) to understand the coverage of cervical cancer prevention and treatment services and provide an opportunity to generate knowledge on risk factors, attitudes and practices extendable globally.

Methods and analysis

This review will be guided by Arksey and O'Malley's framework recommended for conducting scoping review studies. The Preferred Reporting Items for Systematic Review and Meta-Analysis extension for Scoping Reviews (PRISMA-Scr) checklist will also be completed to ensure that the review adheres to sound methodological rigour acceptable for scoping review studies. The following electronic databases will be search for potentially eligible articles: PubMed, Ebsco Host, Scopus and Cochrane Database of Systematic Reviews. Study screening procedures recommended by Higgins and Deeks will be followed.

\section{Discussion}

Cervical cancer can become a disease of the past with a proper control strategy in place. It is therefore imperative to map available evidence on management of cervical cancer to inform policy and advocacy action. More knowledge on the status quo will guide policy makers in ensuring cancer management guiding policies are formulated/updated/ revised accordingly.

Scoping review registration: Not registered with PROSPERO (not needed).

Protocol and registration: This scoping review was not registered.

\section{Background}

Approximately 527600 new CC cases, 265700 deaths were recorded worldwide in 2012 (4). In SSA, CC is the leading cause of cancer deaths among females(5). $t$ is estimated that $90 \%$ of CC deaths occurred in developing countries, 25\% in India (67 500), 60100 deaths in Africa, 144400 in Asia and 28600 in the Caribbean Bay and Latin America (6). Variations in CC rates are due to difference in availability of screening that provide for the detection and treatment of precancerous lesions as well as HPV infection prevalence(7). HPV infection prevalence is highest in Africa (21\%), Latin America and the Caribbean (16\%), Asia (9\%) and North America (5\%) (6). In some Western countries CC rates decreased by $65 \%(6)$. In Norway, CC incidence decreased from 18.7 per 100000 in 1970 to 9.6 per 100000 in 2011 due to well 
established screening programs (6). In USA, the overall cancer death rate dropped by $27 \%$ from 1991 to 2016 whilst in developing countries mortality rates were 2 -fold higher for $\mathrm{CC}$ over the same period (8). In USA CC was the leading cause of cancer death among women in 1930, the death rate was $36 / 100000$, dropped to 5.6 in 1975 and to 2.3 in 2015 due to the development and implementation of PAP test, improved treatment of CC and HPV vaccination with an uptake rate of $47.5 \%$ in 2016 (4). In SSA the greatest threat to $\mathrm{CC}$ management is unavailability of vaccines, information, treatment and monitoring. CC is a neglected area of women's health in LICs (3).

Low-Income Countries recognise the importance of HPV vaccination but getting the resources for the vaccine has been a challenge. Two vaccines, Gardasil and Cervarix are available for protection against two types of HPV that cause approximately $70 \%$ of CC cases (6). The major barrier to the use of the vaccines is their high cost (9). On the other hand, HPV vaccines do not protect against already existing infections and do not protect against all the types of HPV that cause CC (10), therefore screening is a critical component of CC management. Pap test is the most common screening tool for CC in developed countries, while in LICs it is generally inaccessible or met with resistance due to misinformation or poor attitudes (11). Imperative to note here that, a single round HPV testing can reduce the number of CC deaths by about $50 \%(6)$.

Cervical cancer is the mostly screened cancer worldwide(12). Cervical cytology screening programs using Papanicolaou every 3 to 4 years reduced CC incidence and mortality by approximately $80 \%$ in developed countries like North America, Europe, New Zealand, Japan and Australia (13). Screening tests like liquidbased cytology, conventional cytology, HPV testing, visual inspection with acetic acid (VIA), visual inspection with Lugol's iodine (VILI) can detect CIN, if done with quality assurance(14).

Worldwide, 740 women die every day due to CC (15). In Uganda, 5-year age-adjusted standardized survival is as low as 19.8 years (16). HIV-positive people are a high-risk group for HPV infections (17), yet SSA account for over $70 \%$ of global HIV/AIDS burden(18).

HPV testing superiority in reducing mortality from CC was published in 2009 (19), while Australia and Netherlands were the first countries to implement HPV-based screening in their national programs in 2006 (19).

Cervical cancer control is defined as activities to reduce the $\mathrm{CC}$ burden through dissemination and implementation of evidence-based interventions (20). The interventions include prevention, early detection (screening and diagnosis) and treatment (20). Improvements in CC screening, involve addressing systems challenges and changing policies of public health structural interventions which alter structural context for health and are often politicised (21). Women are more likely to seek help when the disease is already at advanced stages (22). Any screening test will have an impact only if it is introduced in a well-planned implemented screening programme (23). Approximately, $90 \%$ of deaths occur in LICs (19), but it is technically possible to control CC globally. 
Prevention of suffering and death from CC is a human rights issue and CC should be given a top priority on the public health agenda. Incidence, survival and death rates can be used to measure progress in CC control with death rates being the best indicator.

\section{Methods}

Scoping review The proposed scoping review will be conducted in accordance with Arksey and O'Malley's (2005) scoping review framework. (24) indicates that the following steps should be undertaken when conducting scoping reviews: (a) identifying the research question; (b) identifying relevant studies; (c) selecting studies; (d) charting the data and (e) collating, summarising and reporting the results. The review will also follow the steps and guidelines outlined in the PRISMA-Extension for Scoping Reviews (PRISMA-ScR) checklist (25) .

\section{Identifying the research question}

The proposed hypothesis for this review is: CC management needs improvement in SSA. The main research question is: What evidence is there on CC management in SSA? The sub research questions include:

- What is the usage/coverage of HPV vaccine in SSA?

- What are barriers to CC screening uptake in SSA?

- At what stage of $\mathrm{CC}$ are most diagnoses made?

- Which drugs are being used for chemotherapy in CC treatment in SSA?

- What is the status of radiotherapy in CC management in SSA?

The aim of this review is to map evidence on CC management in SSA. The objectives of the study are:

- To explore on the coverage/usage of HPV vaccine in SSA;

- To determine the barriers to CC screening uptake in SSA;

- To find out the stages at which most CC is diagnosed in SSA;

- To establish the status of radiotherapy in CC management in SSA and

- To determine the drugs that are being used in $\mathrm{CC}$ treatment.

\section{Identifying relevant studies}

An initial search was performed to determine whether a previous review addressing this topic in SSA was conducted or was in progress. There were no complete or in-process reviews focusing on the coverage of cervical cancer prevention and treatment services in SSA. Based on the review question, the search strategy was developed by identifying the key concepts using the PICO (Problem/Intervention/Comparison/Outcome) approach (26) and further developing the search strategy using controlled vocabulary such as MeSH (Medical Subject Headings) terms. Papers published on CC 
management will be reviewed for each of the following topics: CC prevention, detection (screening and diagnosis) and treatment. A healthcare librarian from the University of Zimbabwe was consulted for the search strategy of electronic databases. The following key search words will be used: CC management or CC control; CC screening or VIAC or PAP smear or HPV testing; CC prevention or HPV vaccine's; CC surgery; $\mathrm{CC}$ treatment or chemotherapy; $\mathrm{CC}$ radiotherapy. African country names and truncated terms such as 'east* Africa' will also be used to ensure that articles indexed using African country specific names or regional terms are retrieved.The operator 'or' will be used to combine synonyms; and the operator 'and' to filter to results which contain all the required terms. Peer Review of Electronic Search Strategies (PRESS) Checklist is used for the search strategy. The databases to be searched include: PubMed, Ebsco Host, Scopus and Cochrane Database of Systematic Review.

\section{Study selection}

Two independent reviewers will conduct abstracts and full article screening. The literature will include published peer-reviewed journal articles with evidence of empirical design utilising either qualitative, quantitative or mixed method research approach addressing the research questions. The screening procedure will be guided by Higgins and Deeks' framework (27). All articles identified to be potentially eligible for inclusion in this review will be obtained in full texts. These articles will be then be exported to reference management software, EndNote version X7. Duplicates will then be removed before further screenings (abstract and full article) are conducted. The PRISMA (Preferred Reporting Items for Systematic Review and Meta-Analysis) flow chart will be used to display screening results of studies (28) (Fig. 1).

\section{Eligibility criteria}

\section{Inclusion criteria}

The inclusion criterion was guided by the following principles to determine articles relevant for this review:

- Studies presenting evidence on cervical cancer

- Studies presenting evidence conducted in SSA

- No limits will be applied for publication date of included studies.

- All study designs will be considered

\section{Exclusion criteria}

Studies that do not focus on humans, as well as those written in languages other than English will be excluded. Non-empirical material like book chapters, opinion papers, commentaries and editorials will also not be included.

\section{Data extraction and charting}


A data collection instrument (Table 1) was developed to confirm study characteristics as well as relevance. Data will be extracted by the principal investigator. The data extraction form will include the following elements: author(s), year of publication, title of study, country, study aim(s) or research question, study design, study setting (urban/rural), study population, sample size, key findings that relate to the review question, study limitations and implications and interpretations and conclusions from the authors. Data will be entered into Access and qualitative data will be uploaded in NVivo, a computer assisted qualitative data analysis software.

\section{Data analysis}

A narrative synthesis will be used, with data synthesised and interpreted using sifting, charting and sorting based on themes, key issues and type of study. Citation tracking will be done using Reference Manager Software in Endnote version X7. Data analysis and tabulation of the findings will be done using Review Manager (RevMan) (29). The narrative synthesis approach (30) will help summarise and identify patterns across studies using tabulations, clustering, textual descriptions, conceptual triangulation (concept mapping) and thematic analysis. Textual data summary will be tabulated from qualitative, mixed methods and quantitative studies. Descriptive statistics will also be used to quantify studies based on the patterns identified. Directed content analysis methods will be used on abstracted data to identify patterns or themes that characterise factors that affect CC management.

\section{Quality control and assessment}

Studies that will be published between the research and report writing will be obtained by subscribing to updates to databases using the search domains used during literature search. Data will be extracted by principal investigator and accuracy will be checked by a second reviewer. Studies with uncertainties about their inclusion will be discussed with a third reviewer.

The quality of evidence will be assessed based on guidance in National Institute for Health and Care Excellence single technology appraisal Specification for Manufacturer/Sponsor Submission of Evidence adapted from the Centre for Reviews and Dissemination's guidance for undertaking reviews in health care (30). Mixed Method Quality Appraisal Tool will be used for quality assurance (13), with aspects like: published in a peer reviewed journal and evidence of sample size calculation will be used to assess quality of included studies. Dissemination of the results will include publications in journals and presentations at health conferences.

\section{Discussion}

Cervical cancer deaths and incidence rates are still high in SSA(31).The rate of control achieved depends on prevention (vaccination and screening) policies. Vaccination of girls has a long term effect on cancer rates because of the long period (1-40 years) (19) from HPV infection to invasive CC, hence control will remain crucial for a long time into the future. 
Global research has shown that among the ways to prevent CC, CC screening and follow-up has the greatest or second greatest impact after vaccination, hence lives can be saved by comprehensive application of available evidence-based interventions to all females who could be affected by CC. The results of the scoping review will be used to inform health policy and knowledge to end users regarding strategies that can be used to facilitate $\mathrm{CC}$ control. The review will be used to identify research gaps that need to be addressed in $\mathrm{CC}$ management. Furthermore, this review will provide a complete and reliable picture of how CC control is being managed in the region, the challenges and opportunities of CC management will be highlighted (32). The results of this review may likely contribute to women's understanding of the relationship between HPV and CC for them to make appropriate, evidence-based decisions on available prevention strategies (33). This scoping review will contribute in informing guidelines regarding $\mathrm{CC}$ control and management.

The outlook for $\mathrm{CC}$ control is bright since the knowledge of what to do is there and the tools to do prophylactic interventions which include, vaccination of the girls, screening and preventive treatment for adult women are well known. Treatment of precancerous lesions is of low cost compared to cost of invasive cancer treatment (which is not readily accessible to many women) (34). It is detrimental to fail to use tools and knowledge for HPV vaccination, screening, and preventive treatment that have saved lives in HICs. Communities need to act urgently to save women and girls from this monster called CC .Cervical cancer is a well-known and preventable cancer and the rates of CC are very varied and are inversely proportional to effectiveness of prevention policies. We could not find mapped evidence on CC management processes to explain the increasing CC deaths and incidence.

\section{Abbreviations}

CC

Cervical Cancer

$\mathrm{CIN}$

Cervical intraepithelial neoplasia

HICs

High Income Countries

HPV

Human Papilloma Virus

LICs

Low Income Countries

LMICs

Low and Middle Income Countries

$\mathrm{MeSH}$

Medical Subject Headings

PAP

Papanicolaou

PICO 
Problem/Intervention/Comparison/Outcome

PRESS

Peer Review of Electronic Search Strategies

PRISMA

Preferred Reporting Items for Systematic Review and Meta-Analysis

PROSPERO

International prospective register of systematic reviews;

RevMan

Review Manager

SSA

Sub-Saharan Africa

VIAC

Visual Inspection with Acetic Acid and Camera

\section{Declarations}

\section{Ethics approval and consent to participate}

Not applicable

\section{Consent for publication}

Not applicable

\section{Availability of data and materials}

All data generated or analyzed during this study will be included in the published systematic review article.

\section{Competing interests}

The authors declare that they have no competing interests.

\section{Funding}

Funding for the scoping review was is applicable, however, the full study for for primary data is funded by the University of KwaZulu-Natal College of health sciences Doctoral research Scholarship grant.

\section{Authors' contributions}

PM conceptualized the study under the supervision of TG, NT and SM and designed data collection methods. $\mathrm{PM}$ and $\mathrm{MH}$ will review the papers for inclusion in the review with disagreements resolved by TG. All authors contributed to the writing and review of the final manuscript. All authors read and approved the final manuscript. 
Acknowledgements

The authors would like to thank the University of KwaZulu-Natal (UKZN) for funding the full study. Standard disclaimer applies.

\section{References}

1. Beral V. Cancer of the cervix: a sexually transmitted infection? The Lancet. 1974;303(7865):1037-40.

2. Nindl I, Rindfleisch K, Lotz B, Schneider A, Dürst M. Uniform distribution of HPV 16 E6 and E7 variants in patients with normal histology, cervical intra-epithelial neoplasia and cervical cancer. International journal of cancer. 1999;82(2):203-7.

3. RATE EA-SM. WHAT IS CERVICAL CANCER? Survival. 1981:64.9.

4. Siegel RL, Jemal A, Wender RC, Gansler T, Ma J, Brawley OW. An assessment of progress in cancer control. CA: a cancer journal for clinicians. 2018;68(5):329-39.

5. Viviano M, DeBeaudrap P, Tebeu P-M, Fouogue JT, Vassilakos P, Petignat P. A review of screening strategies for cervical cancer in human immunodeficiency virus-positive women in sub-Saharan Africa. Int J Womens Health. 2017;9:69.

6. Torre LA, Bray F, Siegel RL, Ferlay J, Lortet-Tieulent J, Jemal A. Global cancer statistics, 2012. CA: a cancer journal for clinicians. 2015;65(2):87-108.

7. Torre LA, Siegel RL, Ward EM, Jemal A. Global cancer incidence and mortality rates and trends-an update. Cancer Epidemiology and Prevention Biomarkers. 2016;25(1):16-27.

8. Piper RJ. NSAMR-How to write a systematic literature review: a guide for medical students. University of Edinburgh 2013.

9. Keating KM, Brewer NT, Gottlieb SL, Liddon N, Ludema C, Smith JS. Potential barriers to HPV vaccine provision among medical practices in an area with high rates of cervical cancer. Journal of Adolescent Health. 2008;43(4):S61-S7.

10. Herweijer E, Sundström K, Ploner A, Uhnoo I, Sparén P, Arnheim-Dahlström L. Quadrivalent HPV vaccine effectiveness against high-grade cervical lesions by age at vaccination: a population-based study. International journal of cancer. 2016;138(12):2867-74.

11. Akinyemiju TF. Socio-economic and health access determinants of breast and cervical cancer screening in low-income countries: analysis of the World Health Survey. PLoS One. 2012;7(11).

12. Vaccarella S, Lortet-Tieulent J, Plummer M, Franceschi S, Bray F. Worldwide trends in cervical cancer incidence: impact of screening against changes in disease risk factors. European journal of cancer. 2013;49(15):3262-73.

13. Piper A. How to write a systematic literature review: a guide for medical students. National AMR, Fostering Medical Research. 2013:1-8.

14. Syrjänen K, Naud P, Derchain S, Roteli-Martins C, Longatto-Filho A, Tatti S, et al. Comparing PAP smear cytology, aided visual inspection, screening colposcopy, cervicography and HPV testing as 
optional screening tools in Latin America. Study design and baseline data of the LAMS study. Anticancer research. 2005;25(5):3469-80.

15. LaVigne AW, Triedman SA, Randall TC, Trimble EL, Viswanathan AN. Cervical cancer in low and middle income countries: addressing barriers to radiotherapy delivery. Gynecologic oncology reports. 2017;22:16-20.

16. Ginsburg 0. Breast and cervical cancer control in low and middle-income countries: Human rights meet sound health policy. Journal of Cancer Policy. 2013;1(3-4):e35-e41.

17. Ursu RG OM, Luca A, Prisecariu LJ, Sălceanu SO, Nemescu D, et al. The Need for Cervical Cancer Control in HIV-Positive and HIV-Negative Women from Romania by Primary Prevention and by Early Detection Using Clinically Validated HPV/DNA Tests. PLoS ONE 10(7):e0132271 doi:101371/journalpone0132271. 2015.

18. Kharsany AB, Karim QA. HIV infection and AIDS in sub-Saharan Africa: current status, challenges and opportunities. The open AIDS journal. 2016;10:34.

19. Nicolas Wentzensen MS. Accelerating cervical cancer control and prevention. Public Health vol320892-9774. 2018.

20. Health WHOR, Organization WH, Diseases WHOC, Promotion H. Comprehensive cervical cancer control: a guide to essential practice: World Health Organization; 2006.

21. Meyerson BE, Zimet GD, Multani GS, Levell C, Lawrence CA, Smith JS. Increasing efforts to reduce cervical Cancer through state-level comprehensive Cancer control planning. Cancer Prevention Research. 2015;8(7):636-41.

22. Moitse jEMKA. Exploration of knowledge of cervical cancer and cervical cancer screening among HIV positive women. curationis. 2014;37(1) Art no 1209.

23. Cruickshank DJ. Cervical cancer screening in developing countries. The journal of the Royal Society for the Promotion of Health. 2005;125(2):95.

24. Arksey H, O'Malley L. Scoping studies: towards a methodological framework. International journal of social research methodology. 2005;8(1):19-32.

25. Tricco AC, Lillie E, Zarin W, O'Brien KK, Colquhoun H, Levac D, et al. PRISMA extension for scoping reviews (PRISMA-ScR): checklist and explanation. Annals of internal medicine. 2018;169(7):467-73.

26. Schardt C, Adams MB, Owens T, Keitz S, Fontelo P. Utilization of the PICO framework to improve searching PubMed for clinical questions. BMC medical informatics and decision making. 2007;7(1):16.

27. Colosia A, Khan S, Hackshaw MD, Oglesby A, Kaye JA, Skolnik JM. A systematic literature review of adverse events associated with systemic treatments used in advanced soft tissue sarcoma. Sarcoma. 2016;2016.

28. Karagiannidou M, Wittenberg R, Landeiro FIT, Park A-L, Fry A, Knapp M, et al. Systematic literature review of methodologies and data sources of existing economic models across the full spectrum of Alzheimer's disease and dementia from apparently healthy through disease progression to end of life care: a systematic review protocol. BMJ open. 2018;8(6):e020638. 
29. Chung AM, Stein MJ, Ghumman A, Zhang J. The effect of post mastectomy radiation therapy on breast reconstruction with and without acellular dermal matrix: a systematic review and metaanalysis protocol. Systematic reviews. 2019;8(1):58.

30. Howell D, Richardson A, May C, Calman L, Fazelzad R, Moradian S, et al. Implementation of selfmanagement support in cancer care and normalization into routine practice: a systematic scoping literature review protocol. Systematic reviews. 2019;8(1):37.

31. De Vuyst H, Alemany L, Lacey C, Chibwesha CJ, Sahasrabuddhe V, Banura C, et al. The burden of human papillomavirus infections and related diseases in sub-saharan Africa. Vaccine. 2013;31:F32F46.

32. Vale C. What is cervical cancer? 1962.

33. Tiro JA, Meissner HI, Kobrin S, Chollette V. What do women in the US know about human papillomavirus and cervical cancer? Cancer Epidemiology and Prevention Biomarkers. 2007;16(2):288-94.

34. Aranda S, Berkley S, Cowal S, Dybul M, Evans T, Iversen K, et al. Ending cervical cancer: A call to action. International Journal of Gynecology \& Obstetrics. 2017;138:4-6.

\section{Table}

Due to technical limitations, Table 1 is provided in the Supplementary Files section.

\section{Supplementary Files}

This is a list of supplementary files associated with this preprint. Click to download.

- PRISMAflowdiagram.docx

- Table1.docx

- Dataextractionform.doc 